\title{
Early Presentation of Hemophagocytic Lymphohistiocytosis Secondary to Severe Dengue: A Case Report
}

\author{
Authors \\ Arun Kumar $\mathbf{N}^{1^{*}}$, Senthil Kumar $\mathrm{SP}^{2}$, Manish Sharma ${ }^{3}$ \\ ${ }^{1}$ Fellow in Paediatric Intensive Care \\ ${ }^{2}$ Consultant Paediatric, Neonatal \& Cardiac Intensivist \\ ${ }^{3}$ Fellow in Pediatric Intensive Care \\ Department of Paediatrics \& Neonatology, Columbia Asia Hospital, Whitefield, Bangalore, Karnataka, India \\ *Corresponding Author

\section{Arun Kumar N}

\begin{abstract}
Background: In recent years increased number of atypical manifestations of dengue fever in children has been reported. Many infections can trigger hemophagocytosis and is often misinterpreted as sepsis with multi-organ dysfunction (MODS). Dengue virus is recognized as a trigger for infection associated hemophagocytosis.

Case Report: A 2 year 11 months old male child presented with history of fever and decreased oral intake since 2 days. Child had signs of severe shock, hepatospleenomegaly with decreased air entry in bilateral lower lung fields on systemic examination. Blood investigations showed thrombocytopenia, leucopenia, negative crp, severely deranged coagulation profile, elevated liver enzymes, dengue NS1 and IgM ELISA positive and high ferritin levels. In view of the above presentation, possibility of secondary hemophagocytic lymphohistiocytosis (HLH) due to dengue infection was considered. Bone marrow aspiration showed histiocytosis with hemophagocytosis. Child met the diagnostic criteria of HLH (2004) and was started on injection dexamethasone according to HLH protocol. Child clinically improved and was discharged on oral dexamethasone.

Conclusion: We would like to emphasise that high level of suspicion is required in diagnosing secondary HLH and it should be considered in the early part of dengue infection if child presents with cytopenias, hyperferritinemia, splenomegaly and MODS.
\end{abstract}

Keywords: Dengue, Hemophagocytic Lymphohistiocytosis, High Ferritin

\section{Introduction}

Dengue fever is a vector borne illness spread through aedesaegypti mosquito, is endemic to India and present with a spectrum of clinical manifestation ranging from self-limiting febrile illness to life threatening shock, severe bleeding manifestation and multi organ failure ${ }^{([1],[2])}$. In recent years, increased number of atypical manifestations of dengue fever have been reported, some of which are severe and associated to with high mortality ${ }^{[3]}$.

Hemophagocytic lymphohistiocytosis (HLH) is an aggressive and life threatening disease due to excessive immune activation. HLH is characterized by excessive secretion of cytokines by the activated macrophages leading to organ failure, dysregulation of natural killer cells and cytotoxic T lymphocytes, 
along with activation and proliferation of histiocytes and uncontrolled hemophagocytosis ${ }^{[4]}$. HLH can be primary, caused by gene mutation or secondary due to autoimmune disease, haematological malignancy and infection, both share a high mortality rate unless appropriate treatment is given ${ }^{[5]}$. Virus-associated hemophagocytic syndrome is characterized by histiocytosis with marked hemophagocytosis in the setting of systemic viral infection which carries high mortality if left untreated. Many infections can trigger hemophagocytosis and is often misinterpreted as sepsis with multiorgan dysfunction which has high mortality. Dengue virus infection is recognized as trigger for virusassociated hemophagocytosis in the recent years ([6], [7]).

We describe a case of 2 years 11 months old child with severe dengue fever, who developed hemophagocytosis on day 3 of illness which is atypical presentation of secondary HLH.

\section{Case Report}

A 2 years 11 month old male child with history of fever since 2 days and decreased oral intake since 2 days, was admitted in a nursing home and was referred to our hospital in view of thrombocytopenia and shock. Child came to our emergency room with shock, on examination child was afebrile, peripheries were cold and cyanosed, hypotensive, low pulse volume and saturation was $92 \%$ in room air. Child had hepatosplenomegaly with decreased air entry in bilateral lower lung fields on systemic examination. Child was resuscitated with IV crystalloids, started on oxygen and other supportive measures. In view of persistent shock child was started on colloids (20\% albumin) and inotropic support. Blood investigations showed thrombocytopenia, leucopenia, negative crp, severely deranged coagulation profile, elevated liver enzymes, dengue NS1 and IgM ELISA positive, elevated LDH(2237 IU/L), normal triglycerides $(57 \mathrm{mg} / \mathrm{dl})$, urea was high $(83 \mathrm{mg} / \mathrm{dl})$,normal creatinine, low fibrinogen level $(154 \mathrm{mg} / \mathrm{dl})$ and high ferritin levels (Table 1). On further investigationsUSG abdomen and chest showed hepatospleenomegaly, massive ascites, B/L pleural effusion and blood culture showed no growth. In view of cytopenias, deranged coagulation profile, splenomegaly and high ferritin levels possibility of secondary HLH due to dengue infection was considered. Bone marrow aspiration showed histiocytosis with hemophagocytosis (Figure 1). Child met the diagnostic criteria of HLH (2004) and was started on injection dexamethasone according to HLH protocol. Chid clinically improved over the next 6days of hospital stay, platelets gradually increased, LFT and coagulation profile normalised, transaminases decreased and child was discharged on tab dexamethasone to continue as per HLH protocol.

Table 1: Trend of Laboratory Results

\begin{tabular}{|l|c|c|c|c|c|c|c|}
\hline Day of Illness & 2 & 3 & 4 & 5 & 6 & 7 & $1^{\text {st }}$ Follow Up \\
\hline Platelet & 24000 & 12000 & 20000 & 27000 & 70000 & 115000 & 148000 \\
\hline Hematocrit (\%) & 40 & 32 & 24 & 21 & 20.4 & 20.0 & 22.6 \\
\hline Hemoglobin (g/dl) & 10.5 & & 7.1 & & & & 7.3 \\
\hline WBC count (cells/mm ${ }^{3}$ ) & & & 8100 & & & & 10200 \\
\hline APTT (sec) & & 54.7 & 47.3 & 37.7 & & 30.9 & \\
PT (sec) & & 23.4 & 21.3 & 16.7 & & 13.4 & \\
INR & & 2.003 & 1.816 & 1.41 & & 1.12 & \\
SGOT (IU/L) & 642 & 1110 & 5091 & 2981 & 1465 & 862 & 95 \\
SGPT ( IU/L) & 226 & 468 & 1808 & 1385 & 1094 & 913 & 499 \\
\hline Ferritin (ng/ml) & & 7587 & & & & & 152 \\
\hline
\end{tabular}




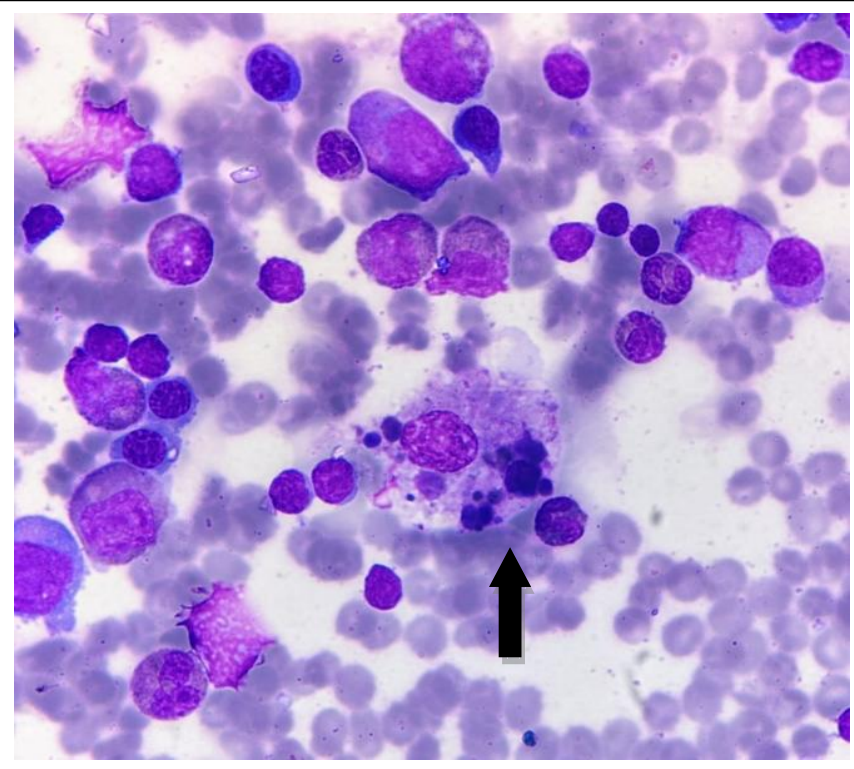

Figure 1: Bone morrow aspiration showing hemophagocytosis, engulfing of lymphocyte and platelets. May Grunwald Giemsa stain, 10X100x”.

\begin{tabular}{l} 
Table 2: The current(2004) diagnostic criteria for \\
HLH are \\
A. Molecular diagnosis consistent with HLH \\
associated mutations (example: PRF1, UNC13D, \\
STX11 etc) OR \\
B. Fulfilment of five out of eight criteria below: \\
1.Fever( $>38.5 *$ C) \\
2.Splenomegaly \\
3.Peripheral blood cytopenia, with at least two of \\
the following: hemoglobin<9g/dl(for infants \\
<4weeks, Hemoglobin <10g/dl); platelets \\
<100,000/microL ; absolute neutrophil count \\
<1000/microL \\
4.Hypertriglyceridemia \\
triglycerides $\quad>265 \mathrm{mg} / \mathrm{dl})$ \\
hypofibrinogenemia (<150mg/dl) and/or \\
5.Hemophagocytosis in bone marrow, spleen, \\
lymph node or liver. \\
6. Low or absent natural killer cell activity. \\
7. Ferritin >500ng/ml \\
8. Elevated soluble CD251: 2 standard deviations \\
above age adjusted laboratory specific norms. \\
\hline
\end{tabular}

\section{Discussion}

Hemophagocytic lymphohistiocytosis is a rare, potentially fatal hyper inflammatory disorder due to excess immune activation and usually presents as prolonged fever and severe sepsis like syndrome ${ }^{[5]}$. Primary HLH (familial HLH) is caused by gene mutation and secondary HLH (sporadic, acquired HLH) is associated with connective tissue disorders, malignancy and infections.
A variety of infections including viral, bacterial, fungal or parasitic can trigger secondary $\mathrm{HLH}^{[6]}$. Most common cause of viral induced secondary HLH is Epstein-Barr virus ${ }^{[8]}$, however one study from India found dengue virus as the most common cause of secondary HLH in India ${ }^{[9]}$. HLH primarily is a pediatric syndrome with infants being commonly affected and highest incidence among infants less than 3 months of age. The male-tofemale ratio is almost 1:1.Although HLH is more common in children, it can affect any age group ${ }^{[10]}$. Earlier literature shows lower incidence rates reflectling many cases being underdiagonised. A older case series from 1970 reported an incidence of 1.2 children per million per year ${ }^{[11]}$ and a another HLH study from Texas pediatric hospital documented an incidence of 1 in hundred thousand children below 18 years $^{[10]}$. A recent case study estimated incidence to be 1 in 3000 admitted children to a pediatric tertiary care hospital which reflects to several cases per center per year ${ }^{[12]}$.

Virus-associated hemophagocytic syndrome presents with non specific clinical features like fever, hepatospleenomegaly and lymphadenopathy with typical laboratory findings such as bicytopenia/pancytopenia, hypertriglyceridemia, hypofibrinogenemia, hepatic impairment with coagulopathy, elevated triglycerides, LDH and ferritin. Diagnosis is confirmed by bone marrow examination demonstrating hemophagocytosis. Due to varied clinical and laboratory findings are associated with HLH, the Histiocytic society has put forth the HLH diagnostic criteria in 1994 which was updated in 2004 (Table 2). According to the criteria, either a molecular diagnosis or 5 out of 8 parameters should be met to arrive at a diagnosis of $\mathrm{HLH}^{[13]}$.

HLH is considered in the differential diagnosis when there is history of prolonged fever (>7days), hepatospleenomegaly and cytopenia ${ }^{[14]}$. High level of suspicion in diagnosing and early institution of treatment may result in good outcome, especially in infection associated HLH where there in in adequate response to general supportive care and whenever a proven definitive therapy is not available for the 
underlying disease process. Our patient is an unusual case where clinical deterioration inspite of general supportive care occurred on day 3 of illness, clinical and laboratory findings prompted us to think of secondary HLH or malignancy. Confirming hemophagocytosis in bone marrow examination and early institution of corticosteroids resulted in clinical improvement of our patient.

\section{Conclusion}

HLH is an aggressive and life threatening disease with high mortality if treatment is delayed. Our patient developed secondary HLH on day 3 of illness corresponding to the early leaky phase of dengue infection which is atypical. We would like to emphasise that high degree of suspicion is required in diagnosing secondary HLH and should be considered in the early part of dengue infection if child presents with cytopenias, hyperferritinemia, splenomegaly and MODS.

\section{Acknowledgment}

We are grateful to Dr .Jyothi, Pediatric Rheumatologist, CAHW and Dr. Sandeep, Dept of Pathology, CAHW for their valuable inputs.

\section{References}

1. Simmons CP, Farrar JJ, Nguyen VC, Wills B. Dengue. N Engl J Med 2012;366:142332.

2. Guzman MG, Harris E. Dengue. Lancet 2015; 385:453-65.

3. Dengue Challenges India's Health System[Internet].http://www.sciencedirect.c om/science/article/pii/S014067361500313X (17 October 2015, last date accessed).

4. Janka GE and Lehmberg K. Hemophagocytic lymphohistiocytosis: pathogenesis and treatment. Hematology Am Soc Hematol Educ Program 2013; 2013: 605-611.

5. Allen $\mathrm{CE}, \mathrm{Yu} \mathrm{X}$, Kozinetz CA and McClai KL. Highly elevated ferritin levels and the diagnosis of hemophagocytic lymphohistiocytosis, Pediatr Blood Cancer 2008; 50(6)1227-1235.

6. Fisman DN. Hemophagocytic syndromes and infection. Emerg Infect Dis 2000;6:6018.

7. Hemophagocytic Syndrome in Severe Dengue Fever: A Rare Presentation [Internet].http://www.ncbi.nlm.nih.gov/pmc/ articles/PMC4192178/ (17 October 2015, date last accessed).

8. Janka G, ZurStadt U. Familial and acquired hemophagocytic lymphohistiocytosis. Hematology Am SocHematol Educ Program. 2005:82-8. [PubMed: 16304363]

9. Ramachandran $\mathrm{B}$, Balasubramanian $\mathrm{S}$, Abhishek N, Ravikumar KG, Ramanan AV. Profile of hemophagocytic lymphohistiocytosis in children in a tertiary care hospital in India. Indian Pediatr. 2011;48:31-5. [PubMed: 20972297]

10. Henter JI, Elinder G, Söder O, Ost A. Incidence in Sweden and clinical features of familial hemophagocytic lymphohistiocytosis. Acta Paediatr Scand 1991; 80:428.

11. Jordan MB, Allen CE, Weitzman S, et al. How I treat hemophagocytic lymphohistiocytosis. Blood 2011; 118:4041.

12. Niece JA, Rogers ZR, Ahmad N, et al. Hemophagocytic lymphohistiocytosis in Texas: observations on ethnicity and race. Pediatr Blood Cancer 2010; 54:424.

13. Bergsten E, Horne A, Aricó M, et al. Confirmed efficacy of etoposide and dexamethasone in HLH treatment: long-term results of the cooperative HLH-2004 study. Blood 2017; 130:2728.

14. Kankananarachchi I, et al." Haemophagocytic Lymphohistuiocytosis (HLH) Secondary to Dengue haemorrhagic fever; A paediatric case series". EC Paediatrics 7.7 (2018): 638-640. 